International Journal of Algebra, Vol. 8, 2014, no. 3, 121 - 128

HIKARI Ltd, www.m-hikari.com

http://dx.doi.org/10.12988/ija.2014.312136

\title{
On Boolean Like Ring Extension of a Group
}

\author{
Dawit Chernet and K. Venkateswarlu \\ Department of Mathematics \\ Addis Ababa University
}

Copyright (c) 2014 Dawit Chernet and K. Venkateswarlu. This is an open access article distributed under the Creative Commons Attribution License, which permits unrestricted use, distribution, and reproduction in any medium, provided the original work is properly cited.

\begin{abstract}
In this paper we introduce the notion of Boolean like ring extension(for short, BLR extension) of a group and obtain various properties. We show that any BLR extension of a group is a group. Further, scalar multiplication on any BLR extension of a group is defined and studied certain properties.
\end{abstract}

Keywords: Boolean like rings, Boolean like ring extension

Mathematics Subject Classificatin: 20K35, 06E99

\section{Introduction}

The concept Boolean Like Rings is due to A.L Foster ([1]).Further studies has been made by Swaminathan V. ([5]). Moreover, Foster also introduced the concept of Boolean extension of a universal algebra ([2]). This paper is a study of BLRextension of a group. This paper is divided in to 4 sections. In section 1, we give some preliminary results on Boolean Like rings and Boolean extension. In section 2, we introduce the notion of BLR extension of a group (Definition 2.1) by generalizing the notion of Boolean extension of a group of Foster A. L [2]. We also proved that the BLR-extension of a group is a group (See Theorem 2.4). Further, we substantiate that the notion of BLR-extension is a proper generalization of Boolean extension of Foster (See example 2.6). In section 3, given any group we identify the group in the BLR-extension. In section 4, we give scalar multiplication on any BLR 
extension of a group and study certain properties. We also introduce norm of an element of a group.

\section{Preliminaries: Boolean Like rings and its prop- erties}

In this section we recollect some definitions and examples concerning the notion of Boolean like rings of A.L.Foster $([1],[2])$, and Swaminathan. V. ([3])

Definition 1.1. A Boolean like ring $R$ is a commutative ring with unity in which for all elements $a, b, \in R, a+a=0, a b(1-a)(1-b)=0$.

Example 1.2. Every Boolean ring is a Boolean like ring but not conversely. For example, the ring $\left(H_{4},+,.\right)$, where $H_{4}=\{0,1, p, q\}$ and the binary operations '+' and '.' defined by

\begin{tabular}{|c|c|c|c|c|}
\hline+ & 0 & 1 & $p$ & $q$ \\
\hline 0 & 0 & 1 & $p$ & $q$ \\
\hline 1 & 1 & 0 & $q$ & $p$ \\
\hline$p$ & $p$ & $q$ & 0 & 1 \\
\hline$q$ & $q$ & $p$ & 1 & 0 \\
\hline
\end{tabular}

\begin{tabular}{|c|c|c|c|c|}
\hline$\cdot$ & 0 & 1 & $p$ & $q$ \\
\hline 0 & 0 & 0 & 0 & 0 \\
\hline 1 & 0 & 1 & $p$ & $q$ \\
\hline$p$ & 0 & $p$ & 0 & $p$ \\
\hline$q$ & 0 & $q$ & $p$ & 1 \\
\hline
\end{tabular}

is a Bolean like ring but not a Boolean ring ( since $p^{2}=0(\neq p)$ ).

Let $\mathrm{R}$ be a Boolean Like ring, then

Lemma 1.3. For all $a \in R, a^{4}=a^{2}$ (weak idempotent law)

Corollary 1.4. For all $a \in R$ and for all non negative integers $n, a^{n+4}=a^{n+2}$.

Corollary 1.5. From corollary 1.4, we have, $a^{n}=a$ or $a^{2}$ or $a^{3}$ for any non negative integer $n$.

Lemma 1.6. In a Boolean like ring $R,\left(\sum_{i=1}^{n} a_{i}\right)^{2}=\sum_{i=1}^{n} a_{i}{ }^{2}$ for all $a_{i} \in R$.

Theorem 1.7. Let $J$ be a Boolean algebra and $U$ be the Boolean extension of an algebra $A$. If $U=U_{[J]}$ is finite(that is if both the kernel $A$ and the core $J$ are finite), the order of $U$ is $n^{k}$ where $n$ is the order of $A$ and $k$ is the number of atoms in $J$.

In the rest of the section $R=(R,+,$.$) is a Boolean Like ring and G=(G,+)$ is a group. 


\section{Boolean Like Ring extension of a Group}

Let $R=(R,+,$.$) be a Boolean like ring and G=(G,+)$ be a group.

Definition 2.1. By a BLR extension of a group, we mean the set $V$ of all mappings $f: G \rightarrow R$ with the following properties:

- P1: $f(x)=0$ except for a finite number of elements $x \in G$;

- P2: $f(x) f(y)=0$ for $x, y \in G$ with $x \neq y$;

- P3: $\sum_{x \in G}(f(x))^{2}=1$

In this article $\mathrm{V}$ denotes the BLR extension of a group $\mathrm{G}$ and an immediate consequence of P2 (of the definition 2.1) of $\mathrm{V}$ is the following:

Lemma 2.2. If $f \in V$ and $f(x)$ is an invertible element of $R$ for some $x \in G$, then $f(y)=0$ for all $y(\neq x) \in R$.

Proof. Let $f \in V$ and $f(x)$ is an invertible element of $\mathrm{R}$ for some $x \in G$. Then, $\exists r \in R$ such that $r f(x)=1$. But then, $f(y)=1 f(y)=r f(x) f(y)=0$ (by P2) for all $y(\neq x) \in R$.

For $f, g \in V$, we define, $f+g$ and $-f$,respectively, as the mapping: $G \rightarrow R$ by:

- $(\mathrm{i}):(f+g)(x)=\sum_{\alpha+\beta=x} f(\alpha) g(\beta)$

- (ii): $(-f)(x)=f(-x)$ for all $x \in G$

Lemma 2.3. If $f, g \in V$, then

$$
\begin{aligned}
& \text { 1. } f+g \in V \\
& \text { 2. }-f \in V
\end{aligned}
$$

Proof. 1. Clearly $f+g$ is well-defined. Now we have to show that $f+g$ satisfies the properties P1, P2, P3. Since $\mathrm{f}(\mathrm{x})$ and $\mathrm{g}(\mathrm{x})$ vanishes for almost all $x \in G$ and $(f+g)(x)$ is defined by the means of equation (i), we have that $(f+g)(x)$ vanishes for almost all $x \in G$. So that $f+g$ satisfies P1. Let $x, y \in G$ and $x \neq y$, then $(f+g)(x)(f+g)(y)=$ $\left(\sum_{\alpha+\beta=x} f(\alpha) g(\beta)\right)\left(\sum_{\gamma+\delta=y} f(\gamma) g(\delta)\right)=\sum_{\alpha+\beta=x} \sum_{\gamma+\delta=y} f(\alpha) g(\beta) f(\gamma) g(\delta)$. Since $x \neq y$, at least one of the conditions $\alpha \neq \gamma$ or $\beta \neq \delta$ must hold. In any case it follows that, $f(\alpha) g(\beta) f(\gamma) g(\delta)=0$. Thus, all the terms in the right hand side of the above equation are zeros. Hence, $(f+g)(x)(f+g)(y)=0$ and $f+g$ satisfies P2. Now 
consider, $\sum_{x \in G}((f+g)(x))^{2}=\sum_{x \in G}\left(\sum_{\alpha+\beta=x} f(\alpha) g(\beta)\right)^{2}=\sum_{x \in G} \sum_{\alpha+\beta=x}(f(\alpha))^{2}(g(\beta))^{2}$ (Since $\mathrm{x}$ runs through all the elements of $\mathrm{G}, \alpha$ and $\beta$ can be made run through all the elements of G. Now, fix $\alpha$ and let $\beta$ run through all the elements of $\mathrm{G}$ ), we have, $\sum_{\alpha \in G}(f(\alpha))^{2}\left[\sum_{\beta \in G}(g(\beta))^{2}\right]=\sum_{\alpha \in G}(f(\alpha))^{2}=1$ (by P3 and Lemma 1.6), showing that $f+g$ satisfies P3. Thus, $f+g \in V$.

2. It is easy to see that $-f$ is in $\mathrm{V}$.

Theorem 2.4. Any BLR extension $V$ of a group is a group.

Proof. It is easy to show that ${ }^{\prime}{ }^{\prime}$ ' defined by (i) is associative. Moreover, The mapping $\tilde{0}: G \rightarrow R$ by $\tilde{0}(0)=1$ and $\tilde{0}(x)=0$ for all $x(\neq 0) \in G$, then $\tilde{0} \in V$ and is the zero for $\mathrm{V}$. For any $f \in V,-f$ defined by (ii) is its inverse.

Remark 2.5. The notion of BLR extension of a group $G$ is a proper generalization to the notion of Boolean-extension of a group G (of Foster ([2])) and we show below by means of an example that this concept is indeed a proper generalization.

Example 2.6. Let $R=Z_{2} \times H_{4}$, then $R$ is a Boolean like ring. In fact, the elements of $R$ are: $a=(0,0), b=(0,1), c=(0, p), d=(0, q), e=(1,0), f=$ $(1,1), g=(1, p)$ and $h=(1, q)$. Let $G$ be the group $\{0,1,2\}$ with addition modulo 3 and $V$ be the BLR extension of $G$. Then any $f \in V$ can be specified by an ordered triple $\{\alpha, \beta, \gamma\}$ where $\alpha, \beta, \gamma \in R$ and $f(0)=\alpha, f(1)=\beta$ and $f(2)=\gamma$. Since $\alpha, \beta, \gamma$ have to satisfy the properties P1, P2, P3 of definition 2, we can verify by direct computation that the elements of $V$ are the only following 18 ordered triplets: $(a, a, f),(a, f, a),(f, a, a),(a, b, e),(a, e, b),(b, a, e),(b, e, a),(e, b, a)$, $(e, a, b),(a, h, a),(a, a, h),(h, a, a),(a, d, e),(a, e, d),(d, e, a),(d, a, e),(e, a, d)$ and $(e, d, a)$.

By theorem 1.7, if $V$ is to be isomorphic with a non trivial Boolean-extension, then the equation $n^{t}=18$ must be solvable in the set of positive integers $n$ and $t(>1)$. Thus, $V$ cannot be isomorphic with any possible Boolean-extension of any group $A$.

\section{Identification of $\mathrm{G}$ in the BLR extension of a group G}

We begin with the following:

Lemma 3.1. For any $g \in G$, Define the mapping $\tilde{g}: G \rightarrow R$ by putting $\tilde{g}(g)=1$ and $\tilde{g}(x)=0$ for all $x(\neq g) \in G$. Then $\tilde{g} \in V$. 
Now define $\tilde{G}=\{\tilde{g}: g \in G\}$.

Theorem 3.2. $\tilde{G}=\{\tilde{g}: g \in G\}$ is a subgroup of $V$.

Proof. Let $g, h \in G$. Then, $(\tilde{g}-\tilde{h})(g-h)=\sum_{\alpha+\beta=g-h} \tilde{g}(\alpha)(-\tilde{h})(\beta)=\sum_{\alpha+\beta=g-h} \tilde{g}(\alpha)(\tilde{h})(-\beta)$

(by $(\mathrm{i})$ and $(\mathrm{ii}))=\tilde{g}(g) \tilde{h}(h)($ by lemma 3.1) $=1$. Thus, $(\tilde{g}-\tilde{h})(x)=0$ for all $x(\neq g-h) \in G($ by lemma 2.2 and 3.1). Thus, $\tilde{g}-\tilde{h}=\overline{(g-h})$ and $\tilde{g}-\tilde{h} \in \tilde{G}$. Hence, $\tilde{G}$ is a subgroup of $\mathrm{V}$.

Theorem 3.3. The mapping $\phi: G \rightarrow \tilde{G}(g \rightarrow \tilde{g})$ is an isomorphism from $G$ to $\tilde{G}$.

Proof. It is clear that the mapping $\phi: G \rightarrow \tilde{G}$ is well- defined and surjective from $G \rightarrow \tilde{G}$. Now, we show that the mapping is injective. Let $g, h \in G$, so that $\phi(g)=\phi(h)$. Then, $\tilde{g}=\tilde{h}$ and hence $\tilde{g}(h)=\tilde{h}(h)=1$ (by definition of $\tilde{h}$ ), so that $g=h$;for other wise $\tilde{g}(h)=0$. Thus, $\phi: G \rightarrow \tilde{G}$ is a one to one mapping. Further, we observe that $\phi(g+h)=\widetilde{(g+h)}=\tilde{g}+\tilde{h}$ (by theorem 3.2) $=\phi(g)+\phi(h)$. Hence, $\phi$ is an isomorphism.

Corollary 3.4. If $V$ is abelian, then $G$ is abelian.

\section{Scalar multiplication in any BLR extension of a group and its properties.}

In this section, we introduce a (scalar) multiplication of the elements of $\mathrm{V}$ by the elements of the $\mathrm{R}$ and study its properties. Further, we define norm of an element of $\mathrm{V}$ and studied its property. Let $a \in R$ and $f \in V$. Then, we define af as the mapping from $\mathrm{G}$ to $\mathrm{R}$ as follows:

(iii) $(a f)(x)=a^{2} f(x)$ if $x(\neq 0) G$ and $(a f)(0)=1-a^{2}+a^{2} f(0)$

Lemma 4.1. For $a \in R$ and $f \in V$, af $\in V$

Proof. Since $f(x)$ vanishes for almost all $\mathrm{x}$ in $\mathrm{G}$, then $a f$ also vanishes for almost all $\mathrm{x}$ in G. Thus, $a f$ satisfies P1. Let $x \neq y$. Then, if both $x, y \neq 0$, then $(a f)(x)(a f)(y)=$ $\left(a^{2} f(x)\right)\left(a^{2} f(y)\right)=a^{2} f(x) f(y)=0$ (by property P2 and Lemma 1.3).If one of them is 0 , (say $x=0$ and $y \neq 0$ ), then $(a f)(0)(a f)(y)=\left(1-a^{2}+a^{2} f(0)\right)\left(a^{2} f(y)\right)$ $=a^{2} f(y)-a^{2} f(y)-a^{2} f(0) f(y)=0$ (by lemma 1.3 and P2) Thus af satisfies the property P2. Now consider, $\sum_{x \in G}((a f)(x))^{2}=((a f)(0))^{2}+\sum_{x \in G, x \neq 0}((a f)(x))^{2}=$ $\left(1-a^{2}+a^{2}(f(0))^{2}\right)^{2}+\sum_{x \in G, x \neq 0} a^{2}(f(x))^{2}=1-a^{2}+a^{2}(f(0))^{2}+\sum_{x \in G, x \neq 0} a^{2}(f(x))^{2}=$ 
$1-a^{2}+a^{2}\left(\sum_{x \in G}(f(x))^{2}\right)=1-a^{2}+a^{2}$ (by P3) $=1$. Thus, af satisfies the property P3 and hence $a f \in V$.

The mapping defined above is called scalar multiplication and have the following properties.

Theorem 4.2. For $a, b \in R$ and $f \in V$

1. $a(b f)=(a b) f$

2. $(a+b) f=a f+b f$ if $a b=0$

3. $a(f+g)=a f+a g$

Proof. 1. If $x \neq 0$, we have $(a(b f))(x)=a^{2}(b f)(x)=a^{2}\left(b^{2} f(x)\right)=\left(a^{2} b^{2}\right) f(x)=(a b)^{2} f(x)$. If $x=0$, we have $(a(b f))(0)=1-a^{2}+a^{2}(b f(0))=1-a^{2}+a^{2}\left(1-b^{2}+b^{2} f(0)\right)=$ $1-a^{2} b^{2}+a^{2} b^{2} f(0)=((a b) f)(0)$. Therefore, $a(b f)=(a b) f$.

2. Let $a, b \in R$ with $a b=0$. If $x \neq 0$, then $((a+b) f)(x)=(a+b)^{2} f(x)=\left(a^{2}+\right.$ $\left.2 a b+b^{2}\right) f(x)=\left(a^{2}+b^{2}\right) f(x)=a^{2} f(x)+b^{2} f(x)$. On the other hand, $(a f+b f)(x)=$ $\sum_{\alpha+\beta=x}(a f)(\alpha)(b f)(\beta)=(a f)(0)(b f)(x)+(a f)(x)(b f)(0)+\sum_{\alpha+\beta=x, \alpha \neq \beta \neq 0}(a f)(\alpha)(b f)(\beta)$ $=\left(1-a^{2}+a^{2} f(0)\right)\left(b^{2} f(x)\right)+\left(a^{2} f(x)\right)\left(1-b^{2}+b^{2} f(0)\right)+\sum_{\alpha+\beta=x, \alpha \neq \beta \neq 0} a^{2} f(\alpha)(\alpha) b^{2} f(\beta=$ $b^{2} f(x)-a^{2} b^{2} f(x)+a^{2} b^{2} f(0) f(x)+a^{2} f(x)-a^{2} b^{2} f(x)+a^{2} b^{2} f(x) f(0)=a^{2} f(x)+b^{2} f(x)$ (By property (P2), we have $f(\alpha) f(\beta)=0$ for every $\alpha \neq \beta$ ). Also, If $x=0$, we have $((a+b) f)(0)=1-(a+b)^{2}+(a+b)^{2} f(0)=1-a^{2}-b^{2}+a^{2} f(0)+b^{2} f(0)$. On the other hand, $(a f+b f)(0)=\sum_{\alpha+\beta=0}(a f)(\alpha)(b f)(\beta)=(a f)(0)(b f)(0)+\sum_{\alpha+\beta=0, \alpha \neq \beta \neq 0}(a f)(\alpha)(b f)(\beta)$ $=\left(1-a^{2}+a^{2} f(0)\right)\left(1-b^{2}+b^{2} f(0)\right)+\sum_{\alpha+\beta=0, \alpha \neq \beta \neq 0} a^{2} f(\alpha) b^{2} f(\beta)=\left(1-a^{2}+a^{2} f(0)\right)(1-$ $\left.b^{2}+b^{2} f(0)\right)$ (Since by (P2) $f(\alpha) f(\beta)=0$ for $\left.\alpha \neq \beta\right)=1-a^{2}-b^{2}+a^{2} f(0)+b^{2} f(0)$ (since $\mathrm{ab}=0)$.

Hence, we have $(a+b) f=a f+b f$ if $a b=0$

3. Let $a \in R$ and $f \in V$. If $x \neq 0,(a(f+g))(x)=a^{2}(f+g)(x)=a^{2} \sum_{\alpha+\beta=x} f(\alpha) g(\beta)$ $=a^{2}\left[f(0) g(x)+f(x) g(0)+\sum_{\alpha+\beta=x, \alpha \neq \beta \neq 0} f(\alpha) g(\beta)\right.$. On the other hand, $(a f+a g)(x)$ $=\sum_{\alpha+\beta=x}(a f)(\alpha)(b f)(\beta)=(a f)(0)(b f)(x)+(a f)(x)(b f)(0)+\sum_{\alpha+\beta=x, \alpha \neq \beta \neq 0} f(\alpha) g(\beta$ 


$$
\begin{aligned}
& =\left(1-a^{2}+a^{2} f(0)\right) a^{2} g(x)+a^{2} f(x)\left(1-a^{2}+a^{2} g(0)\right)+\sum_{\alpha+\beta=x, \alpha \neq \beta \neq 0} a^{4} f(\alpha) g(\beta)= \\
& a^{2} g(x)-a^{4} g(x)+a^{4} f(0) g(x)+a^{2} f(x)-a^{4} f(x)+a^{4} f(x) g(0)+\sum_{\alpha+\beta=x, \alpha \neq \beta \neq 0} a^{4} f(\alpha) g(\beta) \\
& =a^{2} f(0) g(x)+a^{2} f(x) g(0)+\sum_{\alpha+\beta=x, \alpha \neq \beta \neq 0} a^{2} f(\alpha) g(\beta)\left(\operatorname{since} a^{2}=a^{4}\right)=a^{2}[f(0) g(x)+ \\
& f(x) g(0)]+\sum_{\alpha+\beta=x, \alpha \neq \beta \neq 0} a^{2} f(\alpha) g(\beta) . \text { If } x=0,(a(f+g))(0)=1-a^{2}+a^{2}(f+ \\
& g)(0)=1-a^{2}+a^{2}\left[\sum_{\alpha+\beta=0, \alpha \neq \beta \neq 0} f(\alpha) g(\beta)\right] . \text { On the other hand, }(a f+a g)(0)= \\
& \sum_{\alpha+\beta=0}(a f)(\alpha)(b f)(\beta)=(a f)(0)(a g)(0)+\sum_{\alpha+\beta=0, \alpha \neq \beta \neq 0}(a f)(\alpha)(b f)(\beta)=\left(1-a^{2}+a^{2} f(0)\right)(1- \\
& \left.a^{2}+a^{2} g(0)\right)+\sum_{\alpha+\beta=0, \alpha \neq \beta \neq 0}(a f)(\alpha)(b f)(\beta)=1-a^{2}+a^{2} g(0)-a^{2}+a^{4}-a^{4} g(0)+a^{2} f(0)- \\
& a^{4} f(0)+a^{4} f(0) g(0)+\sum_{\alpha+\beta=0, \alpha \neq \beta \neq 0}(a f)(\alpha)(b f)(\beta)=1-a^{2}+a^{2}\left[\sum_{\alpha+\beta=0, \alpha \neq \beta \neq 0}(a f)(\alpha)(b f)(\beta)\right] .
\end{aligned}
$$

Thus, we have, $a(f+g)=a f+a g$.

Corollary 4.3. 1. $a \tilde{0}=\tilde{0}$

2. $0 f=\tilde{0}$ for all $f \in V$

3. $1 f=f$ for all $f \in V$

Proof. 1. Let $a \in R$. If $x \neq 0$, then $(a \tilde{0})(x)=a^{2} \tilde{0}(0)=a^{2} \cdot 0=0$ and If $x=0$, then $(a \tilde{0})(0)=1-a^{2}+a^{2} \tilde{0}(0)=1-a^{2}+a^{2}=1$. Thus, $a \tilde{0}=\tilde{0}$.

2. Let $f \in V$. If $x \neq 0$, then $(0 f)(x)=0^{2} f(x)=0 . f(x)=0$. If $x=0$, then $(0 f)(x)=$ $1-0^{2}+0^{2} f(0)=1$. Thus, $0 f=\tilde{0}$.

3.Let $f \in V$. If $x \neq 0$, then $(1 f)(x)=1^{2} . f(x)=1 . f(x)=f(x)$. If $x=0$, then $(1 f)(0)$ $=1-1^{2}+1^{2} f(0)=1-1+f(0)=f(0)$. Thus, $1 f=f$

Theorem 4.4. Let $V$ be the BLR extension of the a group $G$. Then the mapping $\|\|:. V \rightarrow R(f \rightarrow\|f\|)$ defined by $\|f\|=1-(f(0))$ for any $f \in V$ have the following properties.

1. $\|f\|=0$ if and only if $f=\tilde{0}$.

2. For any $a \in R,\|a f\|=a^{2}\|f\|$

Proof. 1. Suppose $\|f\|=0$. Then, $1-(f(0))=0$. Hence, $f(0)$ is a unit in R. So, $f(x)=0$ if $x(\neq 0) \in G$ (by lemma 2.2). Consequently, $f=\tilde{0}$. Conversely, let $f=\tilde{0}$. Then, $\|f\|=1-(\tilde{0}(0))=1-1=0($ By $(\mathrm{i}))$.

2. Let $a \in R$. Then, $\|a f\|=1-(a f)(0)=1-\left(1-a^{2}+a^{2} f(0)\right)=a^{2}-a^{2} f(0)=$ $a^{2}(1-f(0))=a^{2}\|f\|$ 
Let $\tilde{G}^{*}$ denote the set of all non- zero elements of the subgroup $\tilde{G}$. Then we have the following.

Theorem 4.5. If $\tilde{g}_{1}, \tilde{g}_{2}, \ldots, \tilde{g}_{n} \in \tilde{G}^{*}, a_{1}, a_{2}, \ldots, a_{n} \in R$ with $a_{1}{ }^{2} a_{2}{ }^{2} \ldots a_{n}{ }^{2} \neq 0$ and $a_{1} \tilde{g}_{1}+a_{2} \tilde{g}_{2}+\cdots+a_{n} \tilde{g}_{n}=\tilde{0}$ then $\tilde{g}_{1}+\tilde{g}_{2}+\cdots+\tilde{g}_{n}=\tilde{0}$.

Proof. If possible let $\tilde{g}_{1}+\tilde{g}_{2}+\cdots+\tilde{g}_{n} \neq \tilde{0}$. Then, since $g_{1}+g_{2}+\cdots+g_{n}=0$ implies $\tilde{g}_{1}+\tilde{g}_{2}+\cdots+\tilde{g}_{n}=\tilde{0}$ (by lemma 3.1) and hence $g_{1}+g_{2}+\cdots+g_{n} \neq 0$, so that $g_{1}+g_{2}+\cdots+g_{n}=g$ for some $g(\neq 0) \in G$. Since $a_{1} \tilde{g}_{1}+a_{2} \tilde{g}_{2}+\cdots+a_{n} \tilde{g}_{n}=\tilde{0}$ and $g(\neq 0) \in G$, it follows that, $0=\tilde{0}(g)=\left(a_{1} \tilde{g}_{1}+a_{2} \tilde{g}_{2}+\cdots+a_{n} \tilde{g}_{n}\right)(g)=$ $\sum_{\alpha_{1}+\alpha_{2}+\cdots+\alpha_{n}=0}\left(a_{1} \tilde{g}_{1}\right)\left(\alpha_{1}\right)\left(a_{2} \tilde{g}_{2}\right)\left(\alpha_{2}\right) \cdots\left(a_{n} \tilde{g}_{n}\right)\left(\alpha_{n}\right)$. Now, if $\left(\alpha_{1}, \alpha_{2}, \cdots, \alpha_{n}\right)$ be any other ordered n-tuple other than $\left(g_{1}, g_{2}, \cdots, g_{n}\right)$ such that $\alpha_{1}+\alpha_{2}+\cdots+\alpha_{n}=g=$ $g_{1}+g_{2}+\cdots+g_{n}$, then, $\alpha_{i} \neq g_{i}$ for some i with $\alpha_{i} \neq 0$, so that $\left(a_{i} \tilde{g}_{i}\right)\left(\alpha_{i}\right)=a_{i}{ }^{2} g_{i}\left(\alpha_{i}\right)=0$. This implies that $\left(a_{1} \tilde{g}_{1}\right)\left(\alpha_{1}\right)\left(a_{2} \tilde{g}_{2}\right)\left(\alpha_{2}\right) \cdots\left(a_{n} \tilde{g}_{n}\right)\left(\alpha_{n}\right)=0$. Since, $\tilde{g}_{1}, \tilde{g}_{2}, \ldots, \tilde{g}_{n} \in \tilde{G}^{*}$ and $\tilde{G}^{*}$ consists of the non-zero elements of $\tilde{G}$, it follows that $g_{1}, g_{2}, \ldots, g_{n}$ are all different from 0 in $\mathrm{G}$. And hence it follows that, $0=\tilde{0}(0)=\left(a_{1} \tilde{g}_{1}\right)\left(g_{1}\right)\left(a_{2} \tilde{g}_{2}\right)\left(g_{2}\right) \cdots\left(a_{n} \tilde{g}_{n}\right)\left(g_{n}\right)$ $=a_{1}^{2} a_{2}^{2} \cdot a_{n}^{2}$, which is a contradiction to the hypothesis. Consequently, $\tilde{g}_{1}+\tilde{g}_{2}+$ $\cdots+\tilde{g}_{n}=\tilde{0}$.

Acknowledgements. The first author would like to thank for partial support of ISP(The Swedish International Science Program). We also thank Prof. Eberhard Becker,Dortmund University, Germany for his valuable comments while preparing this paper.

\section{References}

[1] Foster, A.L: The theory of Boolean like rings, Trans. Amer.Math.Soc. Vol.59 (1946),166-187

[2] Foster, A.L: Generalized Boolean theory of universal algebra, math. Zeitschr .Bd.58, S. 306-336 (1953)

[3] Gopala Rao, N.R: Vector space over a regular ring, Math annalen, 167, 280 $-291(1966)$

[4] Swaminathan V: On Fostera's Boolean- like rings, Math. Seminar Notes, Kobe University, Japan, Vol 8, 1980, 347-367.

[5] Swaminathan V: Structure of Boolean Like Rings, Doctoral Dissertation

\section{Received: December 15, 2013}

